\title{
Aufbewahrungsfristen für Aufzeichnungen im Zusammenhang mit der Gewinnung von Blut und Blutbestandteilen, der Herstellung von Blutprodukten und der Anwendung von Blutprodukten
}

\author{
Horst Hasskarl Bita Bakhschai \\ Kanzlei Harms Hasskarl Melzer, Ludwigshafen, Deutschland
}

\section{Schlüsselwörter \\ Archivierungspflichten für Aufzeichnungen . Archivierungsfristen - Adressaten}

\section{Zusammenfassung}

Bei der Gewinnung von Blut und Blutbestandteilen, der Herstellung von Blutprodukten und der Anwendung von Blutprodukten sind zahlreiche gesetzliche und untergesetzliche Verpflichtungen zur Dokumentation und Aufbewahrungsdauer für Aufzeichnungen zu beachten. Adressaten dieser Pflichten sind unter anderem der Arzt, die Blutspendeeinrichtung, der Hersteller, der Zulassungsinhaber (pharmazeutische Unternehmer) oder der Sponsor. In der Praxis hat sich gezeigt, dass es für den betroffenen Adressatenkreis nicht einfach ist, die Archivierungspflichten und die Archivierungsdauer zu ermitteln und ihnen zuverlässig und im erforderlichen Umfang nachzukommen. Nachfolgend wird daher für die betroffenen Adressaten eine praktikable Handhabe zur Ermittlung der wesentlichen Aufbewahrungsfristen für Aufzeichnungen im Blutbereich gegeben. Die wesentlichen Rechtsgrundlagen zur Aufbewahrungsdauer für Dokumente im Blutbereich werden genannt.

\section{Einleitung}

Bei der Gewinnung von Blut und Blutbestandteilen, der Herstellung von Blutprodukten und der Anwendung von Blutprodukten sind zahlreiche gesetzliche und untergesetzliche Verpflichtungen zur Dokumentation und Aufbewahrung der

\author{
Keywords \\ Obligations to archive records - Retention period . \\ Addressees
}

\section{Summary}

Retention Periods for Documentation Relating to the Collection of Blood and Blood Components, the Manufacture of Blood Products and Their Application The collection of blood and blood components, the manufacture of blood products and the application of blood products has to comply with numerous obligations relating to the documentation and the retention period which are based in the law and recommendations. Addressees of these obligations are inter alia the physician, the blood establishment, the manufacturer, the holder of a marketing authorisation (pharmaceutical entrepreneur) or the sponsor. Practical experience has revealed that it is not easy for the concerned addressees to determine and fulfil the obligations to archive and to preserve the retention period in a reliable manner and to the necessary extent. In the following a practical approach is made to compile the essential retention periods relating to documentations in the field of blood. The essential legal basis concerning the retention period of documentations in the field of blood are cited.

Aufzeichnungen $\mathrm{zu}$ beachten. Adressaten dieser Pflichten sind unter anderem der Arzt, die Blutspendeeinrichtung, der Hersteller, der Stufenplanbeauftragte oder eine entsprechend beauftragte Person, der Zulassungsinhaber (pharmazeutische Unternehmer) oder der Sponsor. In der Praxis hat sich gezeigt, dass es für den betroffenen Adressatenkreis nicht ein-

\section{KARGER}

Fax +497614520714

Information@Karger.de

www.karger.com
(C) 2010 S. Karger GmbH, Freiburg 
fach ist, den gesetzlichen und untergesetzlichen Archivierungspflichten zuverlässig und im erforderlichen Umfang nachzukommen, weil die maßgebenden Normen verstreut und in unterschiedlichen Vorschriften enthalten sind. Ein Transparenz verschaffender Überblick und eine Verdeutlichung sind naheliegend und empfehlenswert. Nachfolgend wird daher der Versuch unternommen, für die betroffenen Adressaten eine praktikable Handhabe zur Ermittlung der wesentlichen Aufbewahrungsfristen für Aufzeichnungen im Blutbereich zu geben. Sie ist gegliedert nach Gewinnung von Blut und Blutbestandteilen und Herstellung von Blutprodukten, Anwendung von Blutprodukten und Herstellung und Anwendung von klinischen Prüfpräparaten. Gegenstand dieser Darstellung sind nicht handels- oder steuerrechtliche Aufbewahrungsfristen oder Fristen, die gesetzlich nicht vorgesehen sind, aber sich aus zivilrechtlichen Verjährungsfristen und aus Beweissicherungsgründen ableiten lassen. Gegenstand der Darstellung sind auch nicht Aufbewahrungsfristen für Referenzproben und Rückstellmuster sowie die sicherlich sinnvolle Archivierung der behördlichen Korrespondenz (z.B. Anträge, Mitteilungen, Erlaubnisse, Genehmigungen). Die Richtlinien zur Gewinnung von Blut und Blutbestandteilen und zur Anwendung von Blutprodukten (Hämotherapie) der Bundesärztekammer in der Fassung der Zweiten Richtlinienanpassung 2010 sind berücksichtigt.

\section{Rechtsgrundlagen}

Die wesentlichen Rechtsgrundlagen zur Aufbewahrungsdauer für Dokumente im Blutbereich finden sich in folgenden Vorschriften:

- Arzneimittelgesetz - AMG - (Gesetz über den Verkehr mit Arzneimitteln in der Fassung der Bekanntmachung vom 12. Dezember 2005 (BGBl. I S. 3394), zuletzt geändert durch Verordnung vom 28. September 2009 (BGB1. I S. 3172)), abrufbar unter: www.bundesrecht.juris.de

- Arzneimittel- und Wirkstoffherstellungsverordnung AMWHV - (Verordnung über die Anwendung der Guten Herstellungspraxis bei der Herstellung von Arzneimitteln und Wirkstoffen und über die Anwendung der Guten fachlichen Praxis bei der Herstellung von Produkten menschlicher Herkunft vom 3. November 2006 (BGBl. I S. 2523), zuletzt geändert durch Verordnung vom 26. März 2008 (BGBl. I S. 521)), abrufbar unter: www.bundesrecht.juris.de

- Arzneimittelprüfrichtlinien - AMPrRL - (Zweite Allgemeine Verwaltungsvorschrift zur Änderung der Allgemeinen Verwaltungsvorschrift zur Anwendung der Arzneimittelprüfrichtlinien vom 11. Oktober 2004, BAnz. Nr. 197 vom 16. Oktober 2004), abrufbar unter: www.pei.de

- EG-GMP Leitfaden (Leitfaden für die Gute Herstellungspraxis für Arzneimittel und Prüfpräparate einschließlich seiner Anhänge, BAnz. Nr. 210 vom 9. November 2006, S. 6887), abrufbar unter: www.bmg.bund.de
- (Muster-)Berufsordnung für die deutschen Ärztinnen und Ärzte - MBO-Ä 1997 - (zuletzt geändert durch Beschluss am 24.11.2006; abrufbar unter: www.bundesaerztekammer. de)

- Transfusionsgesetz - TFG - (Gesetz zur Regelung des Transfusionswesens vom 28. August 2007 (BGBl. I S. 2169), zuletzt geändert durch Gesetz vom 17. Juli 2009 (BGBl. I S. 1990)), abrufbar unter: www.bundesrecht.juris.de

- Richtlinien für die Herstellung von Plasma für besondere Zwecke (Hyperimmunplasma) der Bundesärztekammer (1. überarbeitete Fassung 2000, abrufbar unter: $w w w$. bundesaerztekammer.de)

- Richtlinien zur Gewinnung von Blut und Blutbestandteilen und zur Anwendung von Blutprodukten (Hämotherapie) BlutRiLi - (aufgestellt gemäß Transfusionsgesetz von der Bundesärztekammer im Einvernehmen mit dem PaulEhrlich-Institut, in der Fassung der Zweiten Richtlinienanpassung 2010 vom 16. April 2010 (abrufbar unter: $w w w$. bundesaerztekammer.de)

- Richtlinien zur Transplantation peripherer Blutstammzellen (erarbeitet vom wissenschaftlichem Beirat der Bundesärztekammer unter Mitwirkung des Paul-Ehrlich-Instituts, Stand: 01.06.1997; abrufbar unter: www.bundesaerztekammer.de).

\section{Terminologie}

Der gesetzliche und untergesetzliche Normgeber hat für den Blutbereich besondere Begrifflichkeiten definiert. Es ist empfehlenswert, diese Begriffsbestimmungen auch im Rahmen der Frage der Aufbewahrungsdauer der Aufzeichnungen zu beachten.

- Blut (Vollblut) ist das sämtliche nativen Bestandteile enthaltende Blut nach einer Blutspende (Glossar BlutRiLi)

- Blutbestandteile können aus Vollblut mit anschließenden manuellen oder maschinellen Auftrennungsverfahren gewonnen werden oder direkt durch Hämapherese (Glossar BlutRiLi)

- Blutkomponenten sind zelluläre Blutprodukte wie Erythrozytenkonzentrate, Thrombozytenkonzentrate, Granulozytenkonzentrate und therapeutisches Plasma (Glossar BlutRiLi)

- Blutprodukte sind Blutzubereitungen im Sinne des § 4 Abs. 2 AMG, Sera aus menschlichem Blut im Sinne des $\S 4$ Abs. 3 AMG und Blutbestandteile, die zur Herstellung von Wirkstoffen oder Arzneimitteln bestimmt sind ( $\$ 2 \mathrm{Nr}$. 3 TFG)

- Blutspendeeinrichtung ist eine Einrichtung im Sinne von $\S 2$ Nr. 2 TFG, die Blut oder Blutbestandteile entnimmt, testet, verarbeitet, kennzeichnet, verpackt, freigibt, lagert, im Sinne von $\S 4$ Abs. 17 AMG in den Verkehr bringt, einführt, ausführt oder in den oder aus dem Geltungsbereich des AMG verbringt ( $\$ 2$ Nr. 9 AMWHV) 
- Blutzubereitungen sind Arzneimittel, die aus Blut gewonnene Blut-, Plasma- oder Serumkonserven, Blutbestandteile oder Zubereitungen aus Blutbestandteilen sind oder als Wirkstoffe enthalten (§4 Abs. 2 AMG),

- Einrichtung der Krankenversorgung: Krankenhaus oder andere ärztliche Einrichtung, die Personen behandelt ( $\$ 14$ Abs. 2 S. 3 TFG; ausführlich: Glossar BlutRiLi)

- Fertigprodukt ist ein Arzneimittel, dass alle Produktionsstufen, einschließlich der Verpackung in sein endgültiges Behältnis, durchlaufen hat (EG-GMP Leitfaden, Glossar Teil I)

- Klinische Prüfung bei Menschen ist jede am Menschen durchgeführte Untersuchung, die dazu bestimmt ist, klinische oder pharmakologische Wirkungen von Arzneimitteln $\mathrm{zu}$ erforschen oder nachzuweisen oder Nebenwirkungen festzustellen oder die Resorption, die Verteilung, den Stoffwechsel oder die Ausscheidung zu untersuchen, mit dem Ziel, sich von der Unbedenklichkeit oder Wirksamkeit der Arzneimittel zu überzeugen (\$ 4 Abs. 23 AMG)

- Pharmazeutischer Unternehmer ist bei zulassungs- oder registrierungspflichtigen Arzneimitteln der Inhaber der Zulassung oder Registrierung. Pharmazeutischer Unternehmer ist auch, wer Arzneimittel unter seinem Namen in den Verkehr bringt, außer in den Fällen des $§ 9$ Abs. 1 Satz 2 (gemeint: Sponsor) ( $\$ 4$ Abs. 18 AMG)

- Protokolle dokumentieren den Werdegang jeder Charge, einschließlich ihres Vertriebs, sowie alle anderen Sachverhalte, die für die Qualität des Fertigprodukts von Belang sind (Kapitel 4.1 des EG-GMP Leitfadens)

- Prüfpräparate sind Darreichungsformen von Wirkstoffen oder Placebos, die in einer klinischen Prüfung am Menschen getestet oder als Vergleichspräparate verwendet oder zum Erzeugen bestimmter Reaktionen am Menschen eingesetzt werden. Hierzu gehören Arzneimittel, die nicht zugelassen sind, und zugelassene Arzneimittel, wenn diese im Rahmen einer klinischen Prüfung am Menschen in einer anderen als der zugelassenen Darreichungsform oder für ein nicht zugelassenes Anwendungsgebiet oder zum Erhalt zusätzlicher Informationen über das zugelassene Arzneimittel eingesetzt werden ( $\$ 3$ Abs. 3 der Verordnung über die Anwendung der Guten Klinischen Praxis bei der Durchführung von klinischen Prüfungen mit Arzneimitteln zur Anwendung am Menschen, zuletzt geändert durch Verordnung vom 15. März 2006 (BGl. I S. 542))
- Qualität ist die Beschaffenheit eines Arzneimittels, die nach Identität, Gehalt, Reinheit, sonstigen chemischen, physikalischen, biologischen Eigenschaften oder durch das Herstellungsverfahren bestimmt wird ( $\$ 4$ Abs. 15 AMG)

- Spende ist die bei Menschen entnommene Menge an Blut oder Blutbestandteilen, die Wirkstoff oder Arzneimittel ist oder zur Herstellung von Wirkstoffen oder Arzneimitteln und anderen Produkten zur Anwendung bei Menschen bestimmt ist ( $\$ 2$ Nr. 1 TFG)

- Spendeeinrichtung ist eine Einrichtung, die Spenden entnimmt oder deren Tätigkeit auf die Entnahme von Spenden und, soweit diese zur Anwendung bestimmt sind, auf deren Testung, Verarbeitung, Lagerung und das Inverkehrbringen gerichtet ist ( $\$ 2 \mathrm{Nr} .2 \mathrm{TFG}$ )

- Sponsor ist eine natürliche oder juristische Person, die die Verantwortung für die Veranlassung, Organisation und Finanzierung einer klinischen Prüfung bei Menschen übernimmt (§ 4 Abs. 24 AMG).

\section{Aufbewahrungsfristen für die Gewinnung von Blut und Blutbestandteilen und für die Herstellung von Blutprodukten}

Diese Aufbewahrungsfristen sind in Tabelle 1 zusammengefasst.

\section{Aufbewahrungsfristen bei Anwendung von Blutpro- dukten (und von gentechnisch hergestellten Plasma- proteinen zur Behandlung von Hämostasestörungen)}

Diese Aufbewahrungsfristen sind in Tabelle 2 zusammengefasst.

\section{Aufbewahrungsfristen für Herstellung und Anwendung von klinischen Prüfpräparaten}

Diese Aufbewahrungsfristen sind in Tabelle 3 zusammengefasst.

\section{Disclosure}

The authors declared no conflict of interest. 


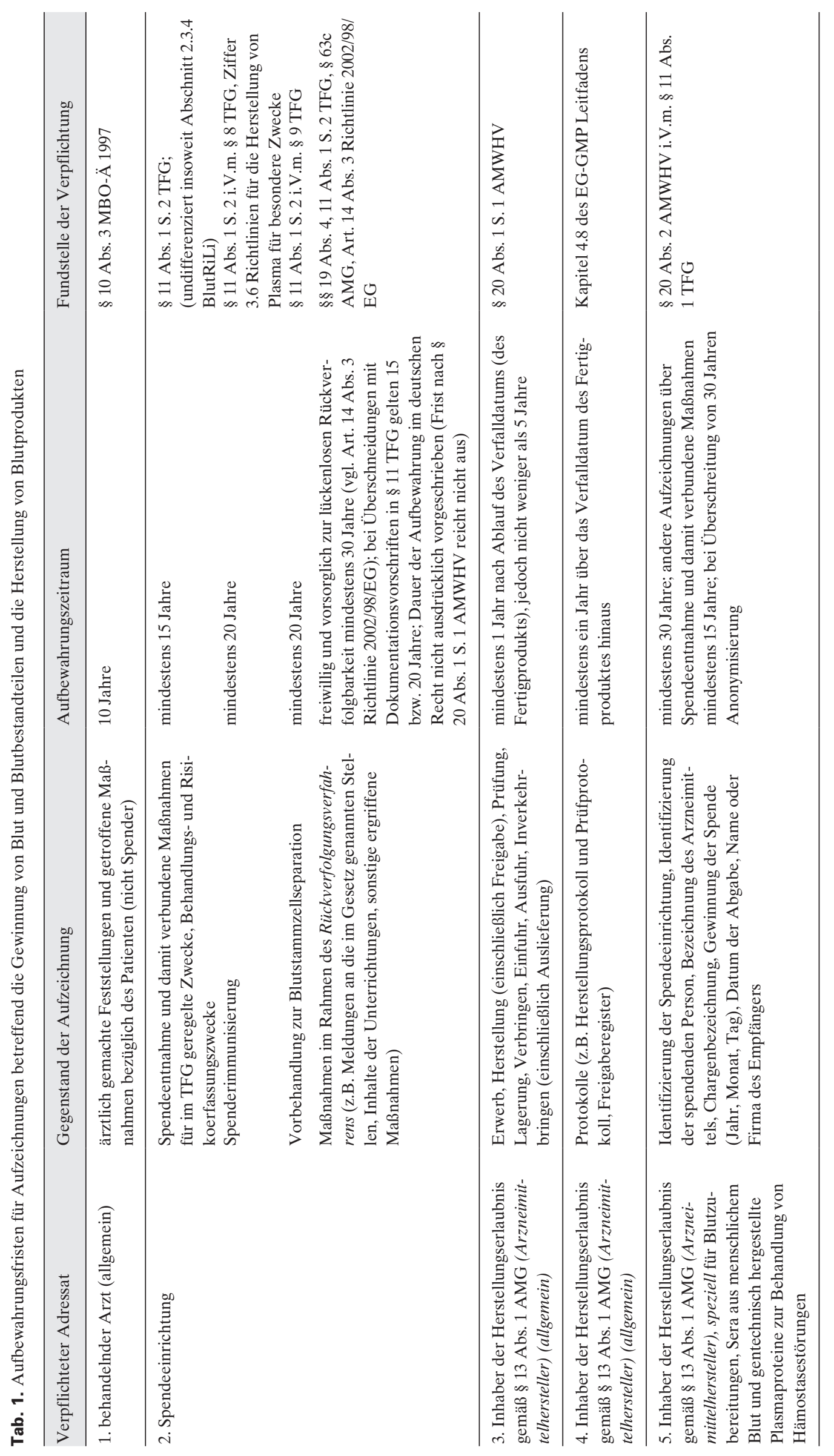




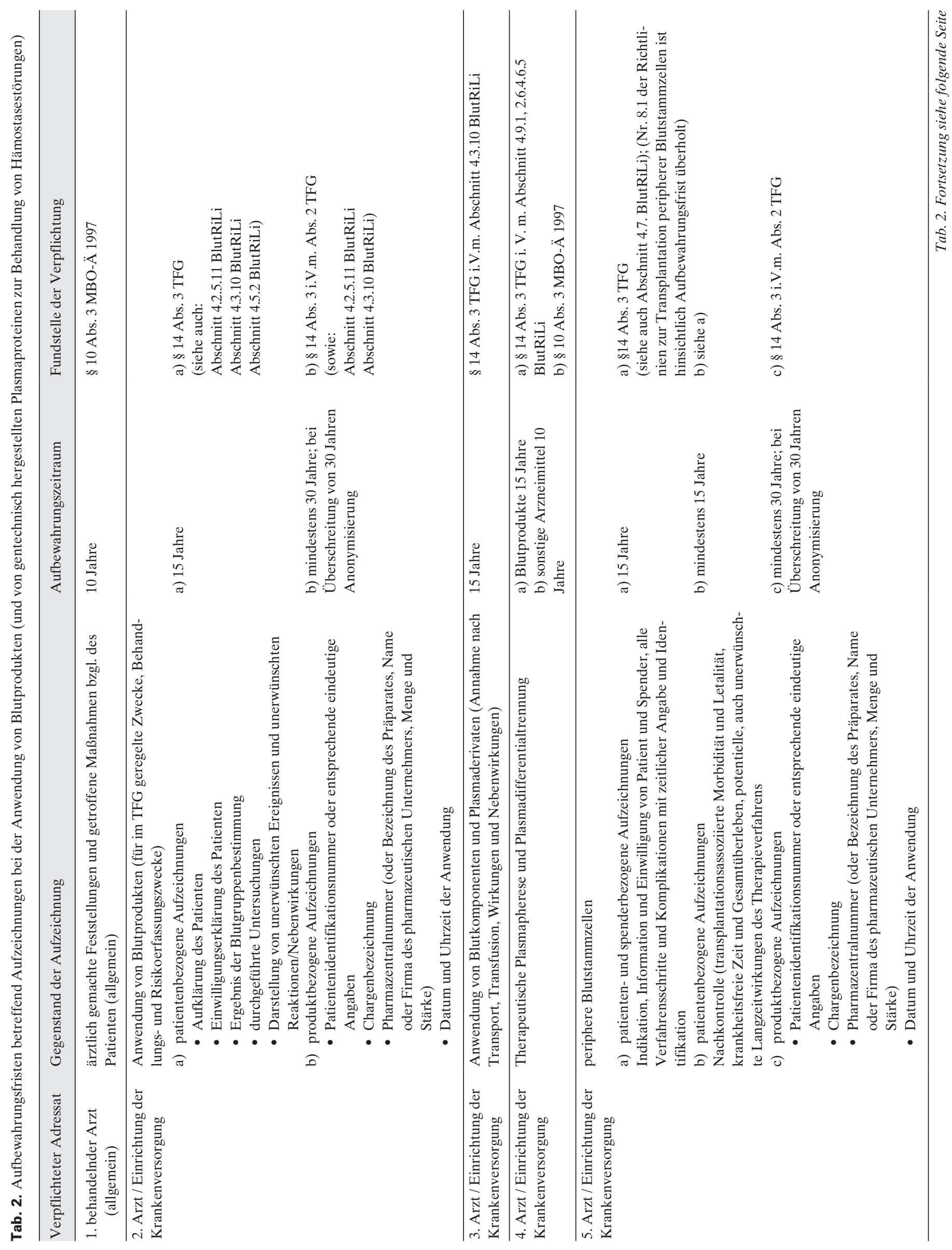




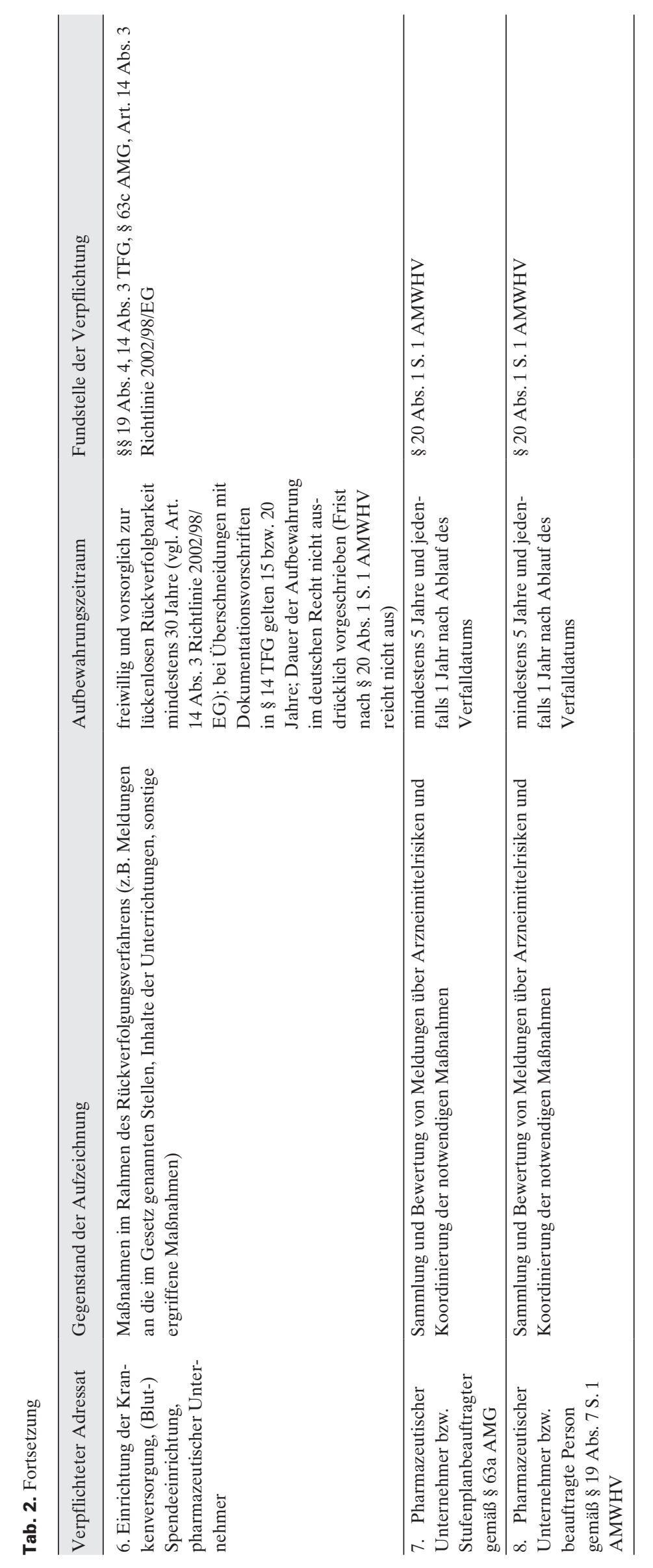




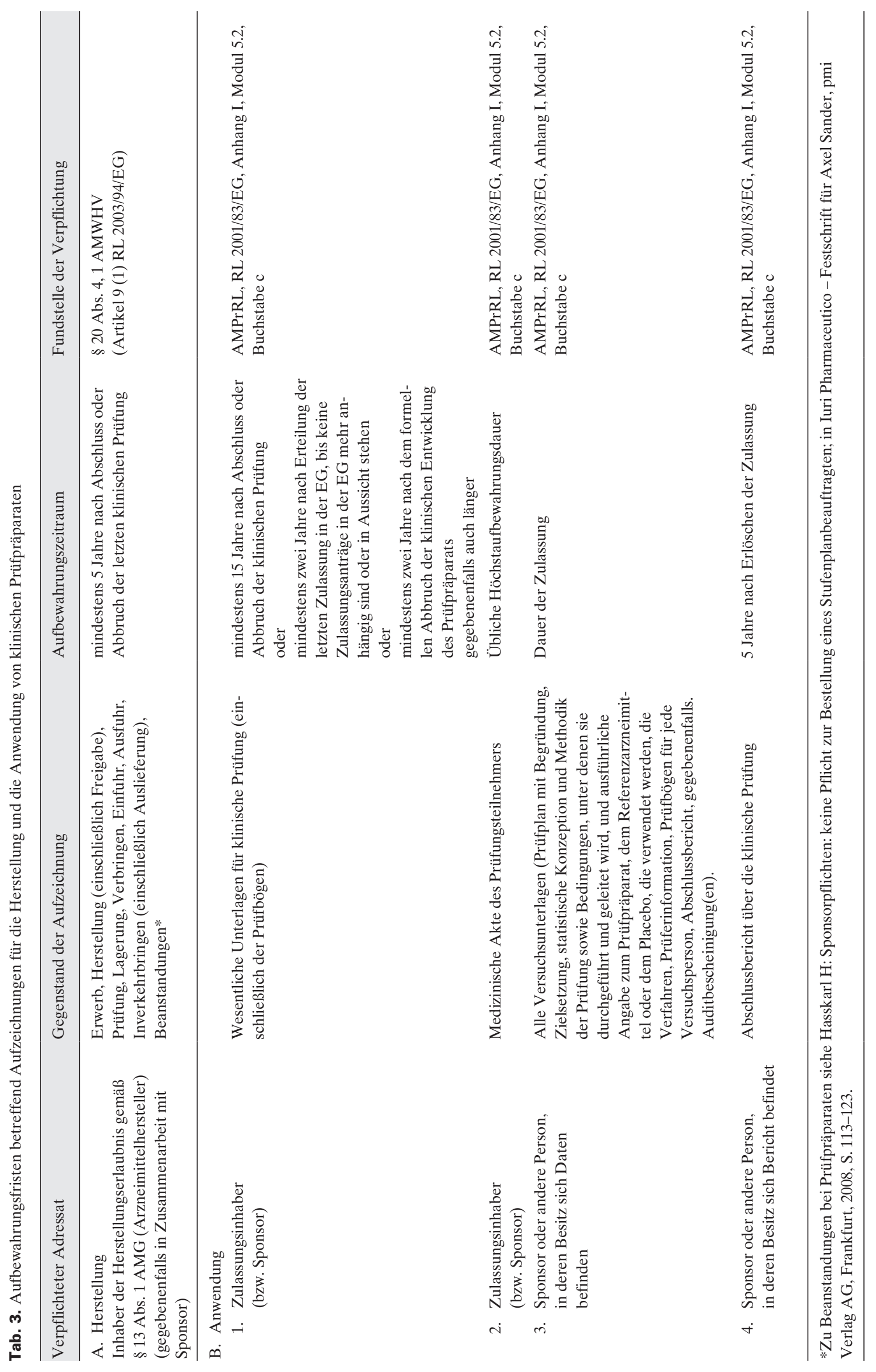

